\title{
Characterizing membrane anchoring of leaf-form ferredoxin-NADP+ oxidoreductase in rice
}

xiaowen $\mathrm{da}^{1}$, jiangfan guo ${ }^{2}$, peng yan ${ }^{1}$, Chao Yang $^{1}$, Hongfei Zhao ${ }^{1}$, yuzhu Kong ${ }^{1}$, ruirui jiang $^{1}$, yi he ${ }^{3}, \mathrm{Xu}$ Jiming$^{1}$, ouyuan $\mathrm{xu}^{4}$, Mao Chuanzao ${ }^{1}$, and xiaorong mo ${ }^{1}$

${ }^{1}$ Zhejiang University State Key Laboratory of Plant Physiology and Biochemistry ${ }^{2}$ Shaanxi Normal University ${ }^{3}$ Zhejiang A and F University State Key Laboratory of Subtropical Silviculture ${ }^{4}$ Zhejiang University

December 12, 2021

\begin{abstract}
Leaf-form ferredoxin-NADP+ oxidoreductases (LFNRs) function in the last step of the photosynthetic electron transport chain, exist as soluble proteins in the chloroplast stroma, and are weakly associated with thylakoids or tightly anchored to chloroplast membranes. Arabidopsis thaliana has two LFNRs, and the chloroplast proteins AtTROL (THYLAKOID RHODANESE-LIKE PROTEIN) and AtTIC62 (62-kDa SUBUNIT OF TRANSLOCON OF INNER CHLOROPLAST MEMBRANE) participate in anchoring AtLFNRs to the thylakoid membrane. By contrast, the membrane anchoring mechanism of rice (Oryza sativa) LFNRs has not been elucidated. Here, we investigated the membrane-anchoring mechanism of LFNRs and its physiological roles in rice. We characterized the rice protein OsTROL1 based on its homology to AtTROL and showed that OsTROL1 is also a thylakoid membrane anchor and its loss led to a compensatory increase in OsTIC62. Moreover, OsLFNR1 attachment through a membrane anchor depends on OsLFNR2, unlike their Arabidopsis counterparts. In addition, OsTIC62 was more highly expressed in rice under dark than under light conditions, consistent with the increased membrane binding of OsLFNR in the dark. Moreover, we observed reciprocal stabilization between OsLFNRs and their membrane anchors. Therefore, our study sheds light on the mechanisms anchoring LFNRs to membranes in rice and highlights differences with Arabidopsis
\end{abstract}

\section{Hosted file}

Characterizing membrane anchoring of leaf-form ferredoxin-NADP+ oxidoreductase in rice.pdf available at https://authorea.com/users/450790/articles/549068-characterizing-membraneanchoring-of-leaf-form-ferredoxin-nadp-oxidoreductase-in-rice 